\title{
EDUCACIÓN AMBIENTAL Y EDUCACIÓN PARA LA CONSERVACIÓN. INICIATIVAS APLICADAS EN LA VEREDA MORTIÑAL DEL MUNICIPIO DE FÓMEQUE, CUNDINAMARCA, COLOMBIA
}

\section{ENVIRONMENTAL EDUCATION AND EDUCATION FOR CONSERVATION. INITIATIVES APPLIED IN THE MORTIÑAL SIDE OF THE MUNICIPALITY OF FÓMEQUE, CUNDINAMARCA, COLOMBIA}

Raúl Iván Clavijo Mejía ${ }^{1}$

Especialista en Educación, Cultura y Política Universidad Nacional Abierta y a Distancia, Bogotá, Colombia

ivanclame@gmail.com

\begin{abstract}
Resumen: En los últimos años la Educación Ambiental -EA- se ha convertido en una herramienta importante en diferentes esferas de la vida social, política y económica, con el fin de introducir en la cotidianidad la preocupación por el cambio climático y la naturaleza. Este artículo busca analizar las experiencias de dos iniciativas de EA en Fómeque, un municipio ubicado al oriente de Bogotá D.C, donde confluyen una iniciativa comunitaria llamada Proyecto La Rana y una iniciativa institucional llamada ECOPARCHE. A partir de la aplicación de entrevistas semiestructuradas y observación participante, se identifica que el Proyecto La Rana se enfoca en una EA que promueve la preservación de los ecosistemas, al tiempo que mantiene formas de ser y hacer de la cultura y producción campesina, teniendo como eje fundamental la comunicación comunitaria y veredal. Por otro lado, el ECOPARCHE desarrolla una EA para la conservación y para la legitimación de la presencia del Parque Nacional Natural Chingaza como autoridad ambiental en la zona. La EA aplicada en contextos rurales colombianos, requiere del posicionamiento de iniciativas a partir del reconocimiento de los saberes populares, del diálogo intergeneracional y del respeto entre los actores que confluyen en el territorio, para así trascender los muros de la escuela y demostrar la funcionalidad de los principios de estas propuestas.
\end{abstract}

Palabras clave: Educación ambiental, conservación, Parque Nacional Natural, campesinos.

Resumo: Nos últimos anos, a Educação Ambiental -EA- tornou-se uma importante ferramenta em diferentes esferas da vida social, política e econômica, a fim de inserir no cotidiano a preocupação com as mudanças climáticas e naturaleza. Este artigo busca analisar as experiências de duas iniciativas de EE em Fómeque, município localizado a leste de Bogotá D.C,

\footnotetext{
${ }^{1}$ Sociólogo de la Universidad Santo Tomás, Bogotá, Colombia
} 
onde convergem uma iniciativa comunitária denominada Projeto La Rana e uma iniciativa institucional denominada ECOPARCHE. A partir da aplicação de entrevistas sem iestruturadas e observação participante, identifica-se que o Projeto La Rana tem como foco uma EA que promova a preservação dos ecossistemas, mantendo modos de ser e fazer da cultura e da produção camponesa, tendo por eixo fundamental comunidade e comunica ção da aldeia. Por outro lado, a ECOPARCHE desenvolve uma EA para a conservação e para a legitimação da presença do Parque Nacional Natural Chingaza como autoridade ambiental na área. A EA aplicada no meio rural colombiano requer o posicionamento de iniciativas baseadas no reconhecimento do saber popular, no diálogo intergeracional e no respeito entre os atores que se unem no território, de forma a transcender os muros da escola e demonstrar a funcionalidade dos princípios dessas propostas.

Palavras-chave: Educação ambiental; conservação: Parque Natural Nacional; agricultores.

Abstract: In recent years, Environmental Education -EA- has become an important tool in different spheres of social, political, and economic life, to introduce concern about climate change and nature into everyday life. This article seeks to analyze the experiences of two EE initiatives in Fómeque, a municipality located east of Bogotá D.C, where a community initiative called La Rana Project and an institutional initiative called ECOPARCHE converge. From the application of semi-structured interviews and participant observation, it is identified that the La Rana Project focuses on an EE that promotes the preservation of ecosystems, while maintaining ways of being and doing of culture and peasant production, having as a fundamental axis community and village communication. On the other hand, the ECOPARCHE develops an EA for the conservation and for the legitimation of the presence of the Chingaza National Natural Park as an environmental authority in the area. EE applied in Colombian rural contexts requires the positioning of initiatives based on the recognition of popular knowledge, intergenerational dialogue and respect between the actors that come together in the territory, to transcend the walls of the school and demonstrate the functionality of the principles of these proposals.

Keywords: Environmental educativo, conservation, National Natural Park, farmers.

\section{INTRODUCCIÓN}

La Vereda Mortiñal se encuentra ubicada en el municipio de Fómeque, Oriente de Cundinamarca, que a su vez hace parte de la Provincia de Oriente, una figura de Ordenamiento Territorial y Administrativo estipulada por el Departamento de Cundinamarca en Colombia. Este municipio tiene una particularidad en comparación con los municipios aledaños, y es que aproximadamente el $31 \%$ de su territorio se encuentra dentro de una zona de especial interés ecosistémico y de conservación estratégica: el Parque Nacional Natural Chingaza (Desde ahora PNNCh).

Esta vereda es una de las más pequeñas del Municipio, sin embargo, cuenta con una escuela rural que integran aproximadamente 15 niños y niñas que cursan desde primero de 
primaria hasta quinto de primaria, y luego se dirigen al colegio municipal donde culminan sus estudios de bachillerato. La actividad productiva de la vereda Mortiñal es esencialmente agrícola y pecuaria, agricultura de subsistencia y en algunas fincas, se cultiva tomate para la comercialización en la zona urbana del municipio y en Bogotá. La estructura de la tenencia de la tierra es de minifundios o fundos medianos, la gran mayoría con títulos de propiedad, casas hechas en material de construcción, cercadas y delimitadas.

La totalidad de habitantes de la vereda son de raíces campesinas del altiplano cundiboyacense, algunos otros con cercanía a los llanos orientales, específicamente Medina y San Juanito, municipios del Departamento del Meta. En su gran mayoría, los procesos de poblamiento en la vereda se dieron a partir de colonizaciones en los años treinta y cuarenta del Siglo XX. Algunos otros llegaron a la zona por cuenta de los trabajos ofrecidos como jornaleros, y unos pocos, en la actualidad, son migrantes venezolanos que han encontrado en las fincas tomateras una oportunidad de vida junto con sus familias. Para el caso particular de la población venezolana, es importante resaltar que es población flotante, se mantienen en la zona un par de meses mientras la subienda tomatera requiere jornaleros y luego algunos migran hacia otros municipios cercanos.

El Parque Nacional Natural Chingaza -PNNCh- se formaliza en 1977 a través de la resolución del Ministerio de Agricultura 154 del 6 de junio de ese año, con un área de 50.000 hectáreas repartidas entre los municipios de La Calera, Fómeque, Guasca, Junín, Choachí y Gachala en el Departamento de Cundinamarca y Medina, el Calvario y Restrepo del Departamento del Meta. En 1978, el Ministerio toma la decisión de ampliar el área en aproximadamente 350 hectáreas adicionales. Este proceso de ampliación finaliza con la resolución 0550 del 19 de junio de 1998 donde se le adjudican al PNNCh 26.000 hectáreas adicionales. La importancia del PNNCh radica en que surte más del $80 \%$ de agua a Bogotá D.C, lo que le hace merecedor de ser reconocido como la fábrica de agua para la capital de Colombia.

Paralelo a este proyecto del Ministerio de Agricultura, la Empresa de Acueducto y Alcantarillado de Bogotá -EAAB- comenzó a desarrollar el Proyecto Chingaza para cumplir con la demanda de agua para Bogotá. El ingreso de la EAAB y del PNNCh al territorio significó no sólo la nueva presencia de actores con poder institucional y de autoridad ambiental, sino además transformó las relaciones de los habitantes que por años vivieron en la región y que tuvieron que salir desplazados por cuenta de una visión del territorio que desconoció los procesos históricos de poblamiento. Al respecto, la Informante 1 resalta que:

Yo tenía una finquita y por allá un acueducto, iban a entrar por allá y entraron con unas cargas por ahí y yo no sabía nada, y cuando supe le dije a mi hijo, si llegan a entrar cargas por ahí no los deje pasar, porque esas son aguas de todos, porque esto no es ni mío es de todos, no pues me amenazaron con la policía, con la autoridad. Esto fue como un mes después de que mi esposo falleciera. Por allá fue y me denunció, una manada de mentiras que era una finca ganadera, piscicultura, agricultura, y yo no tengo nada de eso. (Informante 1, comunicación personal, 2019)

Al dialogar con los informantes clave que tuvieron propiedades en el Páramo de Chingaza, se evidencia un rechazo a la institución. Muchos de ellos se consideran desplazados 
violentamente por parte de miembros del PNNCh o de la EAAB. La delimitación del PNNCh se realizó de manera inconsulta e impuesta, los predios que no contaban con título de posesión, pero si tenían elementos de uso, ocupación y tenencia, fueron reconocidos como baldíos de la nación y adjudicados a las instituciones mencionadas. Este documento presenta unos hechos concretos, como lo son dos iniciativas de EA, desde una interpretación reflexiva que emerge en el terreno y en el ir y venir de la teoría a la observación y trabajo de campo con quienes dinamizan en la vereda los procesos comunitarios e institucionales aquí abordados.

En un momento dado fue una época de desposesión (sic), en algún momento una familia fue dueña casi del todo Páramo, la familia Ricoy ellos tenían hasta 200 reses en el Páramo, yo trabaje allá una semana sembrando papa y la otra arando allá. (Informante 2, comunicación personal, 2019)

Frente a esta situación, la Informante 1 resalta que:

La finca producía nacederos de aguas, buenas aguas, allá quería pasar el acueducto veredal. Llevaron algunas cargas, (dinamita), y poner tanques. Junto con los hijos (sic) se defendieron esos nacimientos, nos acusaron con todas las autoridades, policía, Corpoguavio, Parques Nacionales... [...] No se dejó pasar los equipos, y acusaron falsamente de que la finca tenía producción de marranos y peces. Solo tenía algunas matas de maíz. (Informante 1, comunicación personal, 2019)

El conflicto llegó a escalar al punto de que muchos campesinos quemaban el Páramo de forma indiscriminada y algunas cabañas de los guardaparques también fueron destruidas.

Eso cambió todo, cambió el mundo, esos campesinos no podían seguir desarrollando su vida en ese valle porque todos esos valles eran el objeto de la obra, si fue un quiebre en la historia de las comunidades campesinas, que debían salir de allí, que debieron vender sus tierras y no siempre a un precio justo, no fue la manera como debió ser. (Cuellar, 2012, En: Ramos, 2020, p. 83)

Aunque el PNNCh y la EAAB han adelantado estrategias para reconstruir nuevos lazos comunitarios con las veredas, algunos habitantes siguen guardando recelo ante estas instituciones. Para tratar de menguar el conflicto por uso, ocupación y tenencia de los recursos, se han adelantado iniciativas de Educación Ambiental con enfoque conservacionista y permisos para que los habitantes de los municipios de la zona de influencia del área protegida puedan entrar al Parque sin pagar y con mayores facilidades de acceso.

Paradójicamente, este municipio tiene un déficit de sistema de acueducto y alcantarillado, especialmente en la zona rural, por lo que dicha situación ha sido la dinamizadora de innumerables conflictos con actores estatales como la Empresa de Acueducto y Alcantarillado de Bogotá -EEAB- y el mismo PNNCh por el uso, ocupación y tenencia del agua y de la tierra. Es importante mencionar que el uso productivo de las tierras dentro del PNNCh está restringido para su conservación, y que muchas de las familias que históricamente habitaron el Páramo de Chingaza, fueron víctimas en los años setenta del Siglo XX de desplazamientos por cuenta de una política conservacionista no consensuada con los pobladores, y de una visión centralista del 
ordenamiento territorial que sobrepuso el interés de Bogotá, por sobre el interés de este municipio productor de agua.

Los pobladores no se sienten retribuidos por el agua que brindan a la ciudad de Bogotá y Villavicencio. La riqueza hídrica del municipio es grande, ya que cuenta dentro de su territorio con el Páramo de Chingaza, recolector de grandes cantidades de agua. Este, a su vez, se encuentra bajo la figura institucional de conservación colombiana: Parque Nacional Natural Chingaza. En el municipio se encuentra el embalse de Chuza, el cual abastece el $80 \%$ de agua a los habitantes de Bogotá. La Empresa de Acueducto y Alcantarillado de Bogotá es dueña de gran parte del territorio, en total 17.000 hectáreas del municipio, los cuales también se encuentran enmarcados en predios que pertenecen a la zona en conservación del parque natural. (Ramos Tique, 2020, p. 8)

En este contexto de complejidades simbólicas y de disputas por el uso, ocupación y la tenencia de los recursos, así como de cargas simbólicas sobre la naturaleza, la EA surge como una estrategia con dos esencias: la comunitaria, por un lado, y la institucional por otro. La ubicación geoestratégica de Fómeque hace del municipio un corredor biológico de distintas áreas protegidas que se conectan desde el norte del Departamento de Cundinamarca, hasta el sur del Departamento del Meta, en la región de los Ilanos orientales.

Al analizar el significado de apropiación territorial, es imprescindible comprender de manera inicial qué es el territorio y cómo se construye. Resulta importante entender la forma en que las construcciones simbólicas, las cargas de valor y de creencias se reflejan y materializan en la cotidianidad de las personas en diferentes momentos y contextos. Entender la transición del espacio al territorio, requiere de subjetividades que permiten el reconocimiento del barrio, la vereda, la cuadra o la casa, como parte de la esencia individual y colectiva. En los contextos rurales colombianos, es posible escuchar hablar a múltiples personas al hacer referencia a su parcela como "el terruño", lo que indudablemente denota un cariño, aprecioy apropiación de ese pedazo de tierra que deja de ser un elemento más del espacio, para convertirse en un territorio al que se le otorgan sentimientos, racionalidades y valores en razón de la diaria interacción en dicho campo, donde se construye tejido social y donde se forjan amistades, solidaridades y se garantiza el alimento.

Los procesos de apropiación territorial varían de acuerdo con las condiciones socioeconómicas, políticas y culturales, e incluso se deben tener en cuenta las particularidades propias de cada microestructura en una misma región, en un mismo departamento o en un mismo municipio. La relación de los habitantes con su contexto y los imaginarios que estos construyen de su entorno depende no sólo de las formas en que se poblaron dichos escenarios, sino que obedece a los escenarios futuros deseados, las condiciones de vida aptas para continuar o no en la vereda y las formas en que se presentan cambios en el uso, ocupación y tenencia de la tierra y los recursos. 


\section{LA EDUCACIÓN AMBIENTAL COMO HERRAMIENTA DE TRANSFORMACIÓN Y APROPIACIÓN TERRITORIAL}

Con el inicio del debate y la reflexión en torno al dogma del desarrollo economicista y sus consecuencias en el medio ambiente, repensar las alternativas al desarrollo se convirtió en una imperante necesidad. Dicho proceso de repensar el desarrollo como un dogma sin techo, ha convocado a un sinnúmero de organizaciones gubernamentales y no gubernamentales, comunitarias, barriales, campesinas e indígenas en Colombia, para dar los debates necesarios sobre la importancia de la EA, pero, sobre todo, para qué una EA. A su vez, aparecen en escenarios institucionales propuestas de Educación para la Conservación como una apuesta desde las autoridades ambientales para dar cumplimiento a los Objetivos del Milenio y tiempo después a los Objetivos de Desarrollo Sostenible -ODS-, pues desde esta óptica se debe conservar para garantizar la sostenibilidad ecosistémica y los recursos naturales para proteger la base económica de los países (Caride y Meira, 1998).

\section{La Educación Ambiental como apuesta de cambio social}

Rivarosa y Perales (2006) afirman que uno de los principales retos de la EA, es el análisis de la relación Hombre-Territorio. Así pues, es de suma importancia que la reconfiguración de la relación hombre-territorio esté dada a partir de la transformación de la racionalidad economicista que ha permeado las esferas de la vida pública y privada. En ese sentido, es importante retomar la premisa de Rengifo et al. (2012), quienes consideran que la EA "se debe fundamentar en un cambio de conocimientos y comportamientos de los miembros de la sociedad, en sus relaciones con el medio ambiente lo cual genere una nueva conciencia que provoque una acción cotidiana de protección ambiental" (p. 3).

Las iniciativas de EA encuentran como su principal obstáculo a superar la capacidad de poder integrarse en las actividades cotidianas de los individuos y las comunidades, pero, sobre todo, generar una nueva visión y promover una transformación de las estructuras mentales a partir de la reflexión, debate, análisis y propuestas de alternativas para una sociedad posneoliberal y poscapitalista. Para el caso analizado en este artículo, la EA es fundamental toda vez que "se genera una asimilación de ciencia que nace de los conocimientos cotidianos, de la sistematicidad de uso y de la significación cultural" (Pozo y Gómez, 1998; Rivarosa, 1994 en: Quintana, 2017, p. 929). En tal sentido, las iniciativas de EA que convergen en la Vereda Mortiñal, tienen la posibilidad de influir en el proceso de cambio en la escala axiológica y los imaginarios, las representaciones y los aparatos simbólicos de quienes trascienden del currículo y la teorización hacia la praxis.

El papel de la EA es girar hacia la crítica y construcción de alternativas de desarrollo comunitario y sostenible, en la formación no sólo de ciudadanía que se apropia de su futuro, sino que además se siente participe y se empodera de la protección, cuidado, manejo adecuado y conservación de la naturaleza que le rodea. Por ejemplo, en el estudio de Sánchez y Ossa (2020) en su sistematización de experiencias en cuatro escuelas de la comuna Santa Bárbara, Chile, 
identifican que la enseñanza del impacto de la EA se relaciona con las experiencias de cada estudiante en el aprendizaje dentro del aula, con la aplicación en escenarios verdes de los contenidos del aula y el enfoque pedagógico del docente líder del proceso. Sin embargo, uno de los problemas identificados en este estudio, es el de la continuidad pues si los docentes son cambiados, el ejercicio inicia casi desde cero.

Esta situación ha tratado de evitarse, por ejemplo, en la Institución Educativa Distrital Eduardo Umaña. En la investigación realizada por Carrillo (2018), la autora identifica la necesidad de trascender en el análisis ambiental como reivindicaciones ecológicas y biológicas, a dotar los análisis de elementos identitarios, territoriales y simbólicos que no se quedan en el desarrollo curricular, sino que son a plicados en la cotidianidad y el entorno próximo de los estudiantes. Para el caso analizado por Arredondo et al (2018) en su investigación en escuelas del Estado de Chiapas, México, la aplicación de la EA que trasciende de la práctica pedagógica del aula y es llevada al terreno y al entorno cercano de los estudiantes, está enfocada no sólo en desarrollar los contenidos curriculares, sino en darles continuidad en las prácticas comunitarias. Bernal (2017) en su análisis del impacto de las políticas conservacionistas en Guasca -municipio que también hace parte de la zona de influencia del PNNCh-, hay un especial enfoque en entender que este tipo de políticas han comprendido desde su formulación, al campesino como un depredador que solo extrae recursos. Esta situación ha desconocido la relación histórica de las comunidades y los individuos con la naturaleza, como espacio de creación y fortalecimiento de elementos simbólicos.

La EA en Mortiñal, surge no sólo como una alternativa que permite reflexionar acerca de la importancia biológica del territorio, sino que a su vez permite profundizar en el análisis de la relación hombre-naturaleza. La iniciativa comunitaria, cuenta con tres líneas estratégicas: la EA, la cultura y la comunicación. La primera, entendida como una educación que trasciende las paredes de la escuela y que camina las veredas y reconoce el territorio, pues si se conoce donde se vive, se cuida donde se vive. Reyes y Cardona (2015) mencionan su importancia no sólo en las aulas y en el carácter pedagógico de la formación ambiental, sino además en el proceso de los nuevos enfoques regionales para la planificación en el Ordenamiento Territorial. Es importante mencionar que estos hacen especial énfasis en la EA a nivel regional, en tanto las condiciones biológicas, de biodiversidad y de relacionamiento ecosistémico varían de acuerdo a la posición geográfica, las actividades económicas propias de cada zona y la manera en que sus habitantes se relacionan con su entorno.

\section{La Educación Ambiental para la conservación: ¿una iniciativa desconectada de la realidad?}

La Educación para la Conservación se enmarca como una estrategia de EA con un objetivo claro: posicionar al PNNCh como un actor legítimo en una zona con conflictos socioambientales que datan de los años setenta y ochenta del siglo XX. Este enfoque, afirma González (2002), tiene diferentes perspectivas. Por ejemplo, algunos países asiáticos la definen como las experiencias con la naturaleza y en tal sentido "se habla de una educación en la naturaleza para el desarrollo de las capacidades intelectuales y afectivas" (p. 59). Por otro lado, algunas escuelas estadounidenses enfocan sus propuestas de Educación para la Conservación en la interiorización 
de conceptos científicos para fomentar la apreciación de la belleza de la naturaleza. La Educación para la Conservación del PNNCh junta estos ejemplos en una estrategia que han denominado el ECOPARCHE, donde a través de estrategias de comunicación para compartir conocimientos generados por los profesionales ambientales del mismo, pretenden generar experiencias con la naturaleza con el fin de que, a partir del reconocimiento de las especies de fauna y flora y su belleza, se apropien de la necesidad de conservar el territorio. Esta iniciativa institucional se enfoca en 1. Reconocimiento de flora y fauna. 2. Las aulas vivas y 3. Colegio al parque. A pesar de que es importante no generalizar las iniciativas de Educación para la Conservación como algo que no funciona o algo negativo, González (2002) menciona que "el enfoque dominante en la educación para la conservación es el de educar para conservar individuos, no procesos" (p.79). La iniciativa de conservación del ECOPARCHE dialoga y actúa con base en fundamentos institucionales, epistemológicos y ontológicos de la corriente de los años setenta donde la concepción del ambiente y la naturaleza es la de un recurso y la naturaleza se debe gestionar (Sauvé, 2010).

La conservación implica necesariamente un conocimiento de la ecología, la ciencia que concierne a las relaciones entre la vida y ambiente, pero la ecología misma se basa en una amplia variedad de disciplinas, y la conservación abarca sentimientos, creencias y actitudes a veces complementarias y otras divergentes entre ciencia y tecnología (Odum y Sarmiento, 1998, en: Briceño y Romero, 2007, p. 496)

Es precisamente esta situación la que ha generado una desconexión de las iniciativas institucionales de Educación para la Conservación con los contextos locales y territoriales en los lugares donde se aplica, pues la naturaleza y los ecosistemas no pueden entenderse como elementos aislados de la relación con los seres humanos. Este vacío práctico y ontológico de la conservación lo ha llenado la EA que asume su posición como una iniciativa comunitaria, popular y que comprende los procesos, no los individuos. Esto es importante debatir, en tanto "la conservación es el método de utilización de un recurso natural o el ambiente total de un ecosistema particular, para prevenir la explotación, polución, destrucción o abandono, y asegurar el futuro uso de ese recurso" (Briceño y Romero, 2007, p. 496), y por otro lado las comunidades campesinas tienen sus propias de producción y de conservación bajo sus perspectivas culturales y simbólicas.

El enfoque de la conservación recupera el componente verde del entorno; esto es, pone un mayor acento en problemas ambientales relacionados con lo ecológico, tales como la extinción de especies silvestres y la protección de áreas naturales de interés especial, por su estado de conservación, su representatividad e importancia ecológica o por sus endemismos, aunque también cuando menos eso se observa en algunos de los títulos existe un interés por los recursos naturales (González, 2003, p. 4).

Esto fue abordado en la Conferencia Intergubernamental sobre Educación Ambiental de Tbilisi en 1977, donde se establece que la educación relacionada con la ecología y el medio ambiente debe promover escenarios donde se inculquen conocimientos y valores, pero además donde se cree conciencia sobre la interdependencia social, cultural y política. La Conferencia de Tbilisi identifica la necesidad de una perspectiva holística en la que "se examinen aspectos ecológicos, sociales y culturales y otros que pudieran ser propios de problemas específicos" 
(Conferencia de Tbilisi, 1977, p. 12). A su vez, la Conferencia establece la importancia de que la EA se adapte a la necesidad de los educandos, en su contenido, métodos y materiales específicos, donde la educación no formal -o comunitaria como el caso del Proyecto La Rana-juegan un papel fundamental en tanto son parte de la base comunal y la comunicación social les permite llegar con mayor especificidad a las zonas. Aunque no es posible ubicar la EA en un solo modelo, Novo (2003) establece que el papel del educador ambiental y de la educación ambiental, es ser un mediador en la relación sujeto-contexto, "poniendo en juego las estrategias didácticas necesarias para que las personas implicadas puedan relacionar aquello nuevo que aprenden con lo que ya sabían, bien sea para confirmado y ampliado, o bien para modificarlo o cuestionarlo" (MoreiraSegura et al., 2015, p. 76).

Si bien la EA y la Educación para la Conservación distan en algunos aspectos en términos metodológicos y ontológicos, es innegable que a pesar de buscar objetivos relativamente cercanos con medios que son disímiles, ambas estrategias buscan impactar en el aparato simbólico y valórico de los individuos y las comunidades. Estos marcos de referencia hacen parte de la teoría constructivista que desde la EA se enfoca en abordar el entramado de conocimientos, sentimientos, valores y sensaciones para relacionar lo que sabe y siente (Moreira-Segura et al., 2015). Sin embargo

los programas de EA resultantes de este modelo globalizante se ejecutan mediante la transferencia vertical y unilateral de información culturalmente sesgada, y dirigidos a personas cuyas percepciones y conocimientos propios (personales, tradicionales o de otro tipo) son desacreditados $u$ omitidos y finalmente sustituidos por una visión tecnocrática y economicista de los problemas ambientales y de su solución (Álvarez, 2001, p. 430).

Es en dicha disputa entre globalización y propuestas comunitarias, donde las iniciativas que se analizan en este artículo se encuentran. La EA para la conservación que por sus características es vertical, en muchas ocasiones intenta imponer modelos y prácticas rechazadas por las particularidades socioculturales de los territorios, pues frecuentemente "omiten a la gente y a sus problemas como parte de los componentes del ambiente, manteniendo alienadas a las comunidades de la comprensión de la interdependencia entre la biodiversidad y sus problemas cotidianos" (Shiva, 1993). Por otro lado, la EA posee una racionalidad ambiental que se basa en una escala axiológica donde el ecocentrismo reconoce valores y articula procesos culturales y sustantivos (Leff, 1998). En tal sentido, la racionalidad del Siglo XX y la racionalidad económica se deben debatir, con el fin de generar "procesos de reflexión crítica, de cuestionamientos de la racionalidad económica y homogeneizadora dominante que posibilite a las diversas comunidades legitimar sus saberes frente a los hegemónicos, ponerlos en común, producir y apropiarse de saberes para participar, autogestionar y decidir autónomamente" (García y Priotto, 2009, p. 137).

\section{La apropiación territorial como resultado de la EA}

Los análisis del espacio y su apropiación deben tener en cuenta las características sociopolíticas, históricas, económicas y culturales. Por tal razón, muchos de los procesos de aplicación de EA no han sido del todo fructíferos, pues como se ha mencionado, es inapropiado considerar una sola educación ambiental sin tener en cuenta significados, imaginarios y 
subjetividades que forman la relación hombre-entorno. En el proceso de apropiación del espacio y de implicaciones simbólicas, valóricas e identitarias, se va dando construcción al concepto de territorio. En el proceso de apropiación del espacio y de implicaciones simbólicas, valóricas e identitarias, se va dando construcción al concepto de territorio. "el territorio solo existe en cuanto ya valorizado de múltiples maneras: como zona de refugio, como medio de subsistencia, como fuente de productos y de recursos económicos, como área geopolíticamente estratégica, como circunscripción político-administrativa, como símbolo de identidad" (Giménez, 1996, pp. 10-11). Las iniciativas de EA abordadas en este artículo valorizan al territorio veredal desde diferentes ópticas y perspectivas.

A su vez, al territorio se le otorgan "sentido de pertenencia e identidad, el de conciencia regional, al igual que el ejercicio de la ciudadanía y de acción ciudadana, solo adquieren existencia real a partir de su expresión de territorialidad." (p. 123). Si se concibe la EA como un proceso que fomenta la apropiación territorial, se puede afirmar que esta es una herramienta del ejercicio de territorialidad, en tanto se convierte en un "conjunto de prácticas y sus expresiones materiales y simbólicas capaces de garantizar la apropiación y permanencia de un determinado territorio por un determinado agente social" (Lobato, 1996, en: Gómez y Mahecha, 1998, p. 124). Para el análisis de este artículo el territorio es un espacio de poder en el que las relaciones sociales se materializan, lo que lo hace un escenario móvil, mutable y que se crea y recrea, se transforma, se reconfigura y cambia (Gómez y Mahecha, 1998), y desde luego, uno de esos agentes de cambio y apropiación territorial es la EA.

De la apropiación del territorio se desprenden disputas, negociaciones y normas; mismas que permiten identificar tres grandes entidades interactuantes: la sociedad civil, que da sentido a la noción del interés y el bien público; los grupos o intereses privados asociados, en términos generales, con expectativas de rentabilidad económica y; el estado como entidad que, bajo una perspectiva democrática, emerge de la voluntad ciudadana como conductor y regulador del conjunto de intereses, públicos y privados, mismos que, idealmente, articula bajo un proyecto de nación orientado hacia el desarrollo integral de todos los actores y expresiones sociales (Medina y Guevara, 2018, p. 134).

Por otro lado, Leiva (2020) explica que la apropiación territorial, además de las prácticas de uso, ocupación, tenencia y organización de su entorno, es la creación de redes comerciales, familiares, políticas y culturales. El autor de este documento agregaría a la definición de Leiva sobre apropiación territorial el componente educativo, en tanto el colegio y la escuela además de ser escenarios de garantía de derechos fundamentales, son espacios para la socialización primaria, donde los niños, niñas, jóvenes y adolescentes construyen una serie de conocimientos que deben estar acordes a su contexto. En tanto los programas de EA son apropiados y proyectados con, para y por las comunidades, se genera no sólo un proceso de resignificación con el entorno, sino un proceso de apropiación del territorio, entendido este como la transición de la apropiación del espacio, al que se le asignan cargas simbólicas, de valores y creencias. Un espacio que hace parte extensiva del ser y que se convierte en parte vital de la comunidad, la familia, el individuo o la escuela.

En ese sentido, Tejeda (2002) retoma algunos aspectos relevantes en el estudio de la apropiación territorial: 
1. Las representaciones dadas a partir del uso, ocupación y tenencia de la tierra y de los recursos. Un elemento influenciado por la iniciativa de EA y de Educación para la Conservación en tanto son el resultado de disputas por el control de los recursos.

2. La apropiación a partir de prácticas como la ganadería, la agricultura, la pesca o la cacería. Características propias del ethos y hábitus campesino.

3. Las reglas de primer nivel o la normatividad que hace parte del marco jurídico que determinan el acceso, el uso y la tenencia de los recursos. Parte de los elementos de los que se nutre el ECOPARCHE como apéndice de una autoridad ambiental como el PNNCh.

4. Los mecanismos y las reglas o instituciones informales que hacen parte de la vida veredal y que regulan las relaciones dentro de la esfera microsocial analizada (North, 1993). Eje fundamental que impacta la línea de comunicación del Proyecto La Rana para regular la vida social en el territorio.

5. Los intereses particulares y los conflictos que de ellos emergen, como el ingreso de nuevos actores a la región con objetivos que difieren a las formas de vida habituales (Tejeda y Márquez, 2006). Situación evidenciada en el impacto del ingreso de la EAAB y del PNNCh al territorio, con quienes los campesinos se enfrentaron por el reconocimiento de sus derechos de uso y tenencia de los recursos, así como su derecho de ser reconocido como actor en el territorio.

\section{DISEÑO METODOLÓGICO}

Este artículo es el resultado de una investigación de carácter cualitativo, en tanto implica la utilización de una "amplia variedad de herramientas de recolección de información: entrevistas, historias de vida, grupos focales, trabajo de campo, observación participante y no participante". (Rodríguez, Gil y García, 1996 en: Clavijo, 2019, p. 18). Este artículo analiza las experiencias de dos iniciativas de EA en Fómeque, un municipio ubicado al oriente de Bogotá D.C, donde confluyen una iniciativa comunitaria llamada Proyecto La Rana y una iniciativa institucional en la misma vereda llamada ECOPARCHE. A su vez, en este documento se espera alcanzar una visión holística del contexto estudiado, así como ir más allá de la mera descripción de externalidades, pues es fundamental analizar los puntos de vista de los sujetos a estudiar, así como las condiciones sociohistóricas que permean el desarrollo de las formas de vida (Nolla Cao, 1997). La muestra de este estudio es de casos-tipo (Hernández Sampieri et al., 2014), pues "el objetivo es la riqueza, profundidad y calidad de la información, no la cantidad ni la estandarización" (p. 566).

El enfoque cualitativo puede concebirse como un conjunto de prácticas interpretativas que hacen al mundo "visible", lo transforman y convierten en una serie de representaciones en forma de observaciones, anotaciones, grabaciones y documentos. Es naturalista (porque estudia los fenómenos y seres vivos en sus contextos o ambientes naturales y en su cotidianidad) e interpretativo (pues intenta encontrar sentido a los 
fenómenos en función de los significados que las personas les otorguen). (Hernández Sampieri et al., 1998, p. 42)

El presente estudio privilegia a los actores (Guber, 2001), con el fin de que expresen el sentido de su vida, su cotidianidad, los hechos extraordinarios y su devenir. Para recolectar la información de fuentes primarias se utilizaron el trabajo de campo, la observación participante y la entrevista semiestructurada en la vereda Mortiñal. Entre los meses de julio y diciembre del año 2019 y en los primeros meses del año 2021 se realizaron visitas a campo de una duración aproximada de tres días.

Para el caso de la observación participante, se deben tener en cuenta aspectos como el ambiente físico, el ambiente social y humano, las actividades y los hechos relevantes recordados y remembrados en el proceso de construcción individual y colectiva del territorio. Al respecto, se puede afirmar que los propósitos fundamentales de la observación cualitativa son explorar ambientes, describir contextos comunitarios y comprender procesos a través del tiempo (Hernández Sampieri et al., 2014). La facilidad de la entrevista semiestructurada es que al no ser rígida permite cierta flexibilidad para que el entrevistado mencione o evoque sucesos, retratos o situaciones que podrían haber sido pasadas por alto y que son trascendentales en la investigación, pero a su vez, impide que el entrevistado de otro tipo de información irrelevante para el propósito del estudio.

Díaz Bravo et al (2013), afirman que "en la entrevista semiestructurada, durante la propia situación de entrevista el entrevistador requiere tomar decisiones que implican alto grado de sensibilidad hacia el curso de la entrevista y al entrevistado, con una buena visión de lo que se ha dicho" (p. 164). Entre los meses de julio y diciembre del año 2019 y en los primeros meses del año 2021 se realizaron visitas a campo de una duración aproximada de tres días cada una. En total, las visitas a campo que se realizaron fueron seis, algunas enfocadas exclusivamente en el trabajo de observación participante, y otras enfocadas en el diálogo con los informantes clave y con los funcionarios del PNNCh en donde se aplicaron entrevistas semiestructuradas.

Al plantear un problema en torno a la EA en un contexto donde confluyen dos iniciativas con orígenes disímiles, en una zona de amplia influencia de autoridades ambientales, no puede encasillarse bajo una mirada estática, pues sería desconocer la naturaleza dialéctica y fluctuante de quienes habitan la vereda y de los actores que en ella tienen presencia. Por tal razón, "la acción indagatoria se mueve de manera dinámica en ambos sentidos: entre los hechos y su interpretación, y resulta un proceso más bien "circular" en el que la secuencia no siempre es la misma, pues varía con cada estudio" (Hernández Sampieri et al., 2014, p. 40).

El universo de habitantes de la vereda de aproximadamente 40 -algunos son flotantes o a pesar de vivir en la vereda llevan muy poco tiempo en ella-, pero la muestra tomada para este trabajo es de seis informantes clave, identificados a través de la técnica de la bola de nieve. Estos informantes clave comparten características como haber participado de jornadas de EA de alguna de las dos iniciativas, o haber vivido más de 30 años en el territorio. Sumado a ellos, los informantes clave son los dos miembros dinamizadores del Proyecto La Rana y dos funcionarias del PNNCh que conocieron de cerca la iniciativa del ECOPARCHE. 
La muestra tomada en este documento es diversa, en tanto se busca "mostrar distintas perspectivas y representar la complejidad del fenómeno estudiado, o bien, documentar diversidad para localizar diferencias y coincidencias, patrones y particularidades" (Hernández Sampieri et al. 2014, p. 567). La transcripción de los datos recopilados de la muestra diversa permite identificar dos elementos: el primero es la identificación de categorías que surgen de la experiencia de los individuos -a saber, la EA y la apropiación territorial- y el segundo es la emergencia de relaciones entre conceptos y temas, teniendo como punto de partida la interdependencia de los actores tanto particulares como comunitarios e institucionales, con la Educación Ambiental y la Apropiación Territorial.

Tabla 1. Caracterización de los entrevistados

\begin{tabular}{|c|c|c|c|c|}
\hline Entrevistado & $\begin{array}{ll}\text { Lugar } & \text { de } \\
\text { residencia }\end{array}$ & $\begin{array}{l}\text { Tiempo de } \\
\text { permanencia en } \\
\text { la vereda }\end{array}$ & Género & Ocupación \\
\hline Informante 1 & Vereda Mortiñal & $\begin{array}{l}\text { Desde los años } \\
60\end{array}$ & Femenino & Campesina \\
\hline Informante 2 & Vereda Mortiñal & $\begin{array}{l}\text { Desde los años } \\
60\end{array}$ & Masculino & Campesino \\
\hline Informante 3 & Vereda Mortiñal & Desde el 2010 & Masculino & $\begin{array}{l}\text { Líder del Proyecto La } \\
\text { Rana }\end{array}$ \\
\hline Informante 4 & Vereda Mortiñal & $\begin{array}{l}\text { Desde los años } \\
\qquad 80\end{array}$ & Femenino & $\begin{array}{l}\text { Líder del Proyecto La } \\
\text { Rana }\end{array}$ \\
\hline Informante 5 & Facatativá & N/A & Femenino & $\begin{array}{l}\text { Exfuncionaria del } \\
\text { PNNCh. Línea de } \\
\text { Gobernanza }\end{array}$ \\
\hline Informante 6 & La Calera & N/A & Femenino & $\begin{array}{l}\text { Exfuncionaria del } \\
\text { PNNCh. Línea de } \\
\text { Comunicación y } \\
\text { Educación Ambiental }\end{array}$ \\
\hline
\end{tabular}

Fuente: Elaboración propia.

\section{RESULTADOS}

\section{El Proyecto La Rana: educación ambiental con sentido comunitario}

En el 2016 la iniciativa del Proyecto La Rana se materializa con la identificación de una necesidad: "poner raíces en la vereda con familia y un proyecto digamos más allá de lo que significa la vivienda rural, proyectos ya más estructurados y caminando hacia un desarrollo sostenible" (Informante 3, comunicación personal, 2021). Esta iniciativa es eminentemente comunitaria, y tiene la gran ventaja no sólo de que quienes la dinamizan se encuentran en la vereda y el pueblo de forma permanente, sino porque además hacen parte de una familia que 
habita en la vereda desde los años sesenta. El interés por parte de los miembros del Proyecto La Rana de ejecutar este proyecto en el corto, mediano y largo plazo emerge de:

que hemos decidido tener hijos y los hijos nuestros queremos que sean unos niños transformadores e inspiradores para toda esta dinámica social en torno a lo que hemos gestado desde que estamos acá, y consideramos que nosotros tuvimos la bonita oportunidad de salir a estudiar, de ser profesionales en un ámbito universitario, y nos dijimos que niños como nuestros hijos y los demás hijos de nuestros vecinos, merecen tanto la oportunidad como la que tuvimos nosotros, sabiendo que en el sector rural hay una brecha enorme para acceder a oportunidades de estudiar en la universidad. (Informante 3, comunicación personal, 2021)

El sentido de comunidad que se teje desde el Proyecto La Rana es propio de las formas de vida rural que encuentran en el trabajo comunitario el mejor aliado para lograr objetivos, a diferencia de la hiperindividualización, carácter ontológico de la vida en la ciudad. Para lograr su cometido, los líderes del Proyecto La Rana se mejorar y promover alternativas para que los habitantes de la vereda estuvieran al tanto de las situaciones de su territorio, así nace Ondas de Mortiñal. Este es un grupo de WhatsApp manejado por el Proyecto La Rana que tiene por objetivo:

crear contenidos propios digámonos que atravesándonos a esa agenda noticiosa que manejan los grandes medios en Colombia y lo que manejan los canales en Colombia a través de sus novelas y toda esta cosa, pues hemos creado nuestras propias novelas, nuestras propias radionovelas, nuestros propios documentales, mostrando lo importante que es nuestro territorio a partir de lo que existe: las aves, las demás especies, la cultura propia de un ganadero, de alguien que cultiva el maíz, bueno, mostrando lo propio (Informante 4, 2021).

La Rana cuenta con tres líneas estratégicas: la EA, la cultura y la comunicación. La primera, entendida como una educación que trasciende las paredes de la escuela y que camina las veredas y reconoce el territorio, pues si se conoce donde se vive, se cuida donde se vive. Con la cultura "lo que hacemos es reforzar esa identidad campesina entonces lo que usamos herramientas como rescatar el lenguaje, rescatar las recetas, y alrededor de todo este andamiaje cultural de que el campesino se vea representado en una voz, [...] entonces eso digamos que fortalece mucho el tema del arraigo" (Informante 4, comunicación personal, 2021). Lo que respecta a la comunicación, Ondas de Mortiñal se convirtió en la pieza clave de reproducción de las iniciativas de la vereda y del Proyecto. Esto no sólo permitió posicionar la iniciativa en la vereda como un actor legítimo y con credibilidad, sino que abrió las puertas para que nuevos socios estratégicos llegaran a la vereda: sponsor, donantes, estudiantes universitarios, sindicatos que pagan el internet de la escuela y de la vereda. La comunicación, explican los fundadores del Proyecto, crea tejido y comunidad y potencia la EA y el fortalecimiento de la cultura campesina en la vereda.

El hecho de que el Proyecto La Rana sea una iniciativa de base da paso a abordar de manera más cercana las líneas de acción y su ejecución e impacto en la vereda y sus habitantes, tanto niños como jóvenes y adultos. El objetivo del Proyecto se enmarca en un fundamento teórico, epistemológico y ontológico de la ecología política, en tanto se "establece en ese espacio 
que es el del conflicto por la reapropiación de la naturaleza y de la cultura, allí donde la naturaleza y la cultura resisten a la homologación de valores y procesos (simbólicos, ecológicos, epistemológicos, políticos) inconmensurables y a ser absorbidos en términos de valores de mercado" (Leff, 2003, pp. 11-12).

Es por esto que la iniciativa del Proyecto La Rana se enfoca en los valores simbólicos, ecológicos y culturales. En la labor de materialización de un proyecto para sus hijos y los hijos de los vecinos, hay una disputa por el sentido del territorio, la vereda, el agua, los bosques, la diversidad. Pero también hay una apuesta por reconocer los valores campesinos y de la vida campesina como formas de resistencia ante la avanzada de los valores del neoliberalismo, que ponen un precio a cualquier elemento del entorno y a cualquier persona.

La noción de territorio del Proyecto La Rana, se construye a partir del reconocimiento y conocimiento del entorno y de la importancia de las diferentes especies en el equilibrio ecosistémico. En tal sentido, la EA cumple un papel preponderante al ser la forma en la que se empieza a moldear el territorio como concepto, como imaginario colectivo y como referencia simbólica y de valores. Al comprender al territorio como un todo en el que existe un equilibrio, la relación hombre- naturaleza promovida y entendida desde el Proyecto La Rana es una relación simbiótica. A partir de esta iniciativa se busca entender que el hombre no está en la punta de la cadena alimenticia, sino que hace parte de un todo.

Si un niño de 5 años se enamora de las aves y aprende de ellas no va a cortar un árbol nunca, va a querer sembrar, va a apreciar mejor las flores, va a pensar en la agricultura limpia, va a tener un camino pensando siempre en la sostenibilidad, entonces con este ejercicio que se hace desde La Rana de la educación ambiental, entonces es fundamental para los niños, y por eso entonces (sic) digamos que llegamos a la escuela, poco a poco llegamos a la escuela y hacemos una parte activa (Informante 3, comunicación personal, 2021).

Es el nido, es el nido de todos, es un tejido realmente, un tejido donde estamos todos en ese mundo maravilloso, pues el territorio es ese nido que nos recibe que nos permite transformar, respirar ver crecer a los hijos, pero pues somos parte también de ese tejido (Informante 4, comunicación personal, 2021).

La EA promovida desde el Proyecto La Rana tiene como objetivo trascender la escuela. Si bien es cierto que en la escuela se desarrollan unos contenidos de suma importancia para reconocer el valor biológico y ecológico de la vereda, La Rana tiene como uno de sus principios enseñar a través de los cotidiano y lo práctico, pues al trascender de la teoría a la práctica se tiene un mayor impacto comunitario: "la educación ambiental debe ser una vivencia que debe estar en cada uno de los hogares" (Informante 3, comunicación personal, 2021).

Cuando empezamos a hacer educación en torno a lo que existe, cuando empezamos a mostrar esta mariposa, como se llama, miren este árbol como se llama, miren esta flor como se llama, qué aporte nos da en nuestro ecosistema, por qué son importantes, por qué vale la pena conservarlos. Entonces esa educación ambiental va a hacer sentir a la gente orgullosa del territorio [...]. Eso nos va a enseñar a conservar la especie, los bosques (Informante 4, comunicación personal, 2021). 
El Proyecto La Rana trabaja en la dimensión objetiva y subjetiva de la EA, de la a propiación territorial, en tanto las prácticas de cuidado medioambiental se profundizan a partir del sentido de pertenencia y de importancia de la vereda, de ser de la vereda, de ser campesino. El Proyecto La Rana tiene la visión de la vereda a mediano y largo plazo, como un lugar donde conversan diferentes saberes y donde quienes hoy son niños, tienen la posibilidad de profesionalizarse y de aplicar sus conocimientos para el beneficio de la comunidad.

\section{EI ECOPARCHE: educación para la conservación y la legitimación institucional}

EIECOPARCHE es una iniciativa de EA que surge desde la línea de comunicación del PNNCh en el año 2017 y que no sólo impacta el municipio de Fómeque, sino que intenta abrirse camino e incidir en las comunidades de la zona de impacto del área protegida. El hecho de que el ECOPARCHE sea una iniciativa pensada inicialmente como herramienta de comunicación, da cuenta de que su utilidad se relaciona con compartir y transmitir información del PNNCh y sus actividades, investigaciones y demás elementos. Así pues, esta iniciativa

trata de trabajar digamos (sic) con los niños que hacen parte y están asentados con su familia en la zona de influencia del área protegida, [...] La EA tiene el objetivo de poder construir conjuntamente con los chicos y chicas y de rescatar la importancia que tienen los ecosistemas estratégicos que se encuentran en su territorio (Informante 5, comunicación personal, 2021).

EI ECOPARCHE es una herramienta con objetivos mucho más locales de conservación. Desde luego, esta iniciativa no puede desligarse del discurso institucional de la conservación que por años ha convertido a Parques Nacionales Naturales en un referente nacional del cuidado del medio ambiente. En ese sentido, uno de los objetivos del ECOPARCHE es precisamente fomentar la valoración social de la biodiversidad en las áreas protegidas:

mediante la construcción de conocimientos y el rescate de saberes locales en torno a un tema de conservación especifico, y poderlos transformar en un lenguaje fácil de entender para las comunidades, a través de ejercicios audiovisuales y radiales, poder comunicar una información clara, que sea factible de recordar a corto, mediano, largo plazo por las comunidades y que estas se sientan identificadas, ese es el objetivo (Informante 5, comunicación personal, 2021).

Si bien es cierto es importante conservar, no se puede separar del territorio la relación hombre-naturaleza. Por tal razón, en algunas ocasiones el ECOPARCHE y en términos generales el PNNCh no son considerados actores con legitimidad, en tanto muchos campesinos ven que esta institución al ser una autoridad ambiental limita los procesos productivos propios de la vida campesina y que incluso fue generadora de desplazamiento, como han mencionado algunos entrevistados. Sin embargo, para tratar de mitigar esta situación y esta carga simbólica que por años ha tenido que llevar el PNNCh, desde el ECOPARCHE se genera:

un proceso de construcción colectiva y además que el nivel de organización de ellos es bastante importante, pues digamos característica que no se puede evidenciar en todos los territorios donde pues el parque tiene jurisdicción, pero digamos este elemento de 
organización social es muy importante para que estos procesos de educación ambiental posean y hagan mella, dentro de la importancia de la conservación de esta biodiversidad (Informante 5, comunicación personal, 2021).

Sobre las formas de incidencia en Mortiñal, existen tres líneas de acción: 1. Reconocimiento de flora y fauna. 2. Las aulas vivas y 3. Colegio al parque. La primera línea de acción establece la necesidad de que dentro de la EA los habitantes de la vereda cuenten con los conocimientos básicos de biología para conservar y además "es poder identificar cuáles han sido esos usos tradicionales que se le ha dado desde las comunidades tanto a la flora como a la fauna existente en el territorio (Chingaza)" (Informante 5, comunicación personal, 2021). Las aulas vivas como segundo pilar del ECOPARCHE tienen como objetivo generar "visitas de niños, niñas, jóvenes al área protegida para que ellos desde lo práctico puedan evidenciar la importancia de estos ecosistemas estratégicos" (Informante 6, comunicación personal, 2021). Esta iniciativa pretende convertir en reproductores de conocimiento y conciencia ambiental a los núcleos familiares.

En la tercera línea de acción del ECOPARCHE se encuentra la comunicación, que se enfoca en la creación de contenido documental para divulgar investigaciones y socializar con los habitantes de las veredas, las acciones llevadas por el PNNCh. Este pilar del ECOPARCHE, surge de generar un ambiente propicio para la conservación y protección de un ecosistema que provee el agua para Bogotá. Es por eso que muchos de los habitantes de las veredas, consideran que la actuación del PNNCh y la EAAB no contribuye a la justicia ambiental y sí profundiza conflictos ecológicos distributivos, en tanto los servicios ambientales están enfocados a cubrir la demanda de agua de Bogotá.

En lo que respecta al territorio, desde el ECOPARCHE se entiende como parte de un proceso de conservación, pero que de cierta manera intenta traducirse en un lugar con una amalgama de actores con objetivos y fines que pueden diferir o ser similares. Esta visión de territorio desde el ECOPARCHE ha ido transformándose desde la práctica, al comprender que el territorio no es un espacio que contiene elementos aptos para garantizar servicios ecosistémicos y que deben conservarse, sino que va más allá de criterios materiales hasta lo subjetivo:

En este caso se entiende al ECOPARCHE como un proceso que se vale de técnicas comunicativas para poder llevar un mensaje claro y concreto en torno a la conservación y acciones en torno a la conservación que se pueden llevar desde lo local y lo regional (Informante 5, comunicación personal, 2021).

Se le considera no sólo como un espacio físico, sino como esa posibilidad de generar el intercambio de relaciones, también de relaciones de poder donde transcurren un millón de lógicas teniendo en cuenta pues que este territorio es un área protegida que tiene ecosistemas estratégicos, bastante importantes a nivel por ejemplo de regulación en el suministro de agua. (Informante 6, comunicación personal, 2021).

El discurso de la conservación es una característica central de todas las iniciativas que desde el PNNCh se adelantan, y el ECOPARCHE no es una excepción. A pesar de que entienden al territorio como un lugar donde conviven diferentes actores con distintos intereses, el ECOPARCHE busca posicionar el discurso de la conservación desde sus formas de incidencia y en su población 
objetivo, sin embargo, un proceso crítico y de reflexión dentro de la institución misma ha permitido identificar diferentes acciones encaminadas a tratar de limar asperezas con las comunidades. De tal manera, el ECOPARCHE se convierte en una estrategia de construcción de lazos y de confianza, con el objetivo trazado en la conservación de especies y ecosistemas, a partir de la gobernanza que sólo se logra con el trabajo mutuo y el reconocimiento del otro -para este caso las comunidades campesinas-, como un actor válido, que no es nuevo en el territorio y que cuenta con unas prácticas ancestrales que son el sustento para sus familias.

\section{CONCLUSIONES}

El origen de la Iniciativa del Proyecto La Rana es comunitario, una familia que vuelve a la vereda para establecer una familia y desde allí ser replicadores del conocimiento para transformar realidades de la zona a partir de unos elementos clave como la comunicación. Así pues, el objetivo de la iniciativa de EA del Proyecto La Rana se enfoca no sólo en conservar ciertos ecosistemas, sino además en promover el uso responsable de los elementos que la naturaleza brinda. La noción de recursos no cabe dentro del carácter ontológico del Proyecto La Rana, en tanto su fin es además de la protección del ecosistema local, reivindicar la cultura campesina y construir tejido social en el territorio y con las diferentes comunidades.

Por su parte, el ECOPARCHE es una iniciativa evidentemente institucional, que, aunque coincide con el Proyecto La Rana en la importancia de conservar, su discurso ortodoxo y rígido limita el horizonte de la iniciativa. Desde luego, se debe reconocer que una iniciativa de PNN no puede desligarse de la política institucional, pero sería muy útil el hecho de que el ECOPARCHE fuera algo más allá de una propuesta para introducir al PNNCh como un actor legítimo en las comunidades. Se podría incluso afirmar que consolidar el ECOPARCHE y sus líneas de acción no es un fin en sí, sino un medio para abrirle camino a Parques Nacionales Naturales de Colombia PNN-.

Uno de los resultados más importantes de este artículo es identificar que a pesar de tener objetivos comunes, las iniciativas que confluyen en la Vereda Mortiñal difieren en las formas y el fondo de sus acciones y actividades. El simple hecho de que para el ECOPARCHE no se dialoguen términos de Educación Ambiental sino de Educación para la Conservación, demarca sustancialmente el cómo, el porqué y el para qué de la iniciativa ambiental. Además de esto, es de suma importancia resaltar que el ECOPARCHE es parte de la línea de acción de comunicación, lo que da paso a entender que esta es una herramienta de reproducción de lo que se hace dentro del parque en términos de conservación y cuidado del área protegida.

A su vez, el ECOPARCHE emerge como una propuesta no sólo de comunicación, sino también como representante ante las comunidades de un actor altamente rechazado por cuenta de situaciones del pasado como el desplazamiento, la aplicación de comparendos ambientales, las sanciones a diferentes personas y la transformación en el uso, ocupación y tenencia de los recursos de una región históricamente habitada por campesinos con costumbres y particularidades propias de un pueblo del oriente de Cundinamarca. Por su parte, la EA del Proyecto La Rana se enfoca en discutir y reflexionar acerca de la importancia de la naturaleza en una relación bidireccional entre las comunidades y su entorno. La mirada campesina y la 
experiencia del día a día del trabajo en la vereda y en las fincas del Proyecto La Rana, permite identificar que esta iniciativa no sólo tiene la posibilidad de ser más duradera en el tiempo, sino que su impacto es mayor en tanto son una familia de la comunidad los dinamizadores de esta iniciativa. Ahora bien, la EA propuesta desde el Proyecto La Rana es integral, entiende que los proyectos de vida de los niños y niñas dependen de las oportunidades, y la EA de La Rana pretende garantizar algunas de las oportunidades.

Ambas iniciativas coinciden en que le territorio es una construcción social, eso es innegable. El territorio es escenario de disputa, pero también es donde los valores, las creencias, la espiritualidad y las cargas simbólicas se materializan. En esa lógica, el territorio es concebido como un lugar del que los habitantes de la Vereda Mortiñal deben apropiarse, pero no sólo de manera física en términos de la delimitación de predios y de la propiedad privada, sino de comprender que, en palabras de los líderes del Proyecto La Rana, están ahí de paso y es un lugar prestado.

El territorio es comprendido como el lugar donde se tejen las relaciones vecinales y donde se desarrollan las actividades productivas, pero como se entiende desde el ECOPARCHE y desde el Proyecto La Rana, debe guardar unas condiciones óptimas de un equilibrio ecosistémico, que sólo se logra a partir del reconocimiento de la importancia de las diferentes especies en ese ciclo vital y a su vez, del proceso de apropiación territorial de la vereda como punto de socialización de los diferentes actores de las diferentes edades. Desde luego, el ECOPARCHE intenta introducir en el imaginario colectivo que el PNNCh hace parte del territorio y que no es un actor externo que en los años setenta cometió cierto tipo de acciones contra campesinos de la región. Ese sin lugar a duda, es una de las mayores diferencias al hablar del territorio, pues el ECOPARCHE mantiene su enfoque de proteger y cuidar los servicios ecosistémicos para una ciudad como Bogotá, una mirada centralista que se mantiene desde la época de llegada del Parque al territorio. 


\section{REFERENCIAS BIBLIOGRÁFICAS}

Álvarez, A. (2001). De la herencia cotidiana al tesoro perdido: nuevos desafíos en la educación ambiental para la conservación de la biodiversidad. Interciencia, 26 (10), 429-433. ISSN: 0378-1844.

Arredondo M, Saldívar, A, y Limón, F. (2018). Estrategias educativas para abordar lo ambiental. Experiencias en escuelas de educación básica en Chiapas. Innovación educativa (México, DF), 18(76), 13-37.

Bernal, H. (2017). Implicaciones de las políticas de conservación ambiental en la relación de los habitantes de Guasca con su entorno. Revista de antropología iberoamericana, 12 (3), 365- 387.

Briceño, H. y Romero, R. (2007). Formación de valores en educación ambiental para la conservación del ecosistema. Telos, 3 (9), 491-508. ISSN: 1317-0570.

Caride, J. y Meira P. (1998). Educación ambiental y desarrollo: La sustentabilidad y lo comunitario como alternativas. Pedagogía Social, 2, 7-30. Universidad Santiago de Compostela.

Carrillo, L. (2018). La formación de los jóvenes en liderazgo ambiental como un factor de apropiación territorial: el caso de la IED Eduardo Umaña Mendoza. Nodos y Nudos, 42 (5), 51- 71. ISSN: 0122-4328ISSN-E: 2619-6069pp. 51-71.

Conferencia de Tbilisi. (1977). Conferencia intergubernamental sobre Educación Ambiental. Organizado por la UNESCO con la cooperación de PNUMA. Recuperado de: https://www.minam.gob.pe/cidea7/documentos/Declaracion-de-Tbilisi-1977.pdf.

Díaz-Bravo, L., Torruco-García, U., Martínez-Hernández, M., \& Varela Ruiz, M. (2013). La entrevista, recurso flexible y dinámico. Investigación en educación médica, 2(7), 162-167.

García, D., y Priotto, G. (2009). Educación ambiental. Buenos Aires: Jefatura de Gabinete de Ministros, Secretaría de Ambiente y Desarrollo Sustentable.

Giménez, G. (1996). Estudios sobre las culturas contemporáneas, Territorio y cultura, 2(4), 9-30.

Gómez, G. y Mahecha, O. (1998). Espacio, territorio y región: conceptos básicos para un proyecto nacional. Cuadernos de geografía: Revista colombiana de geografía, 7(1-2), 120-134.

González, E. (2002). Educación ambiental para la biodiversidad: reflexiones sobre conceptos y prácticas. Tópicos en Educación Ambiental, 4 (11), 76-85.

González, E. (2003). Atisbando la construcción conceptual de la educación ambiental en México. Consejo Mexicano de Investigación Educativa. Recuperado de: http://ww.anea.org.mx/docs/Gonzalez-AtisbandoFinalCOMIE.pdf.

Hernández, R., Collado, C. F., Lucio, P. B., Valencia, S. M., y Torres, C. P. M. (2014). Metodología de la investigación. Mcgraw-hill. México, DF. 
Leiva, A. (2020). "El medio ambiente: la empresa más grande del mundo". Reconfiguraciones sociales de la institucionalidad ambiental en el Islote, Colombia. Territorios, (42-Especial), 1-31. Doi: https://www.doi.org/10.12804/revistas.urosario.edu.co/territorios/a.7737.

Leff, E. (1998). SABER AMBIENTAL. Sustentabilidad, racionalidad, complejidad, poder. Siglo Veintiuno Editores. ISBN: 968-23-2141-7. México.

Medina, L. y Guevara, F. (2018). Apropiación territorial y recursos hídricos en la cuenca de los ríos Grijalva y Usumacinta, México. Agua y territorio, 12, 133-144. DOI 10.17561/at.12.3505.

Moreira-Segura, C., Araya-Rodríguez, F. y Charpentier-Esquivel, C. (2015). Educación ambiental para la conservación del recurso hídrico a partir del análisis estadístico de sus variables. Revista Tecnología en Marcha, 28(3), 74-85.

Novo, M. (2003). La educación ambiental. Editorial Universitas. Madrid, España.

North, Douglas. (1993). Instituciones. Recuperado de: http://ebour.com.ar/pdfs/Instituciones,\%20de\%20D ouglass\%20 North. pdf.

Quintana-Arias, R. F. (2017). La educación ambiental y su importancia en la relación sustentable: Hombre- Naturaleza-Territorio. Revista Latinoamericana de Ciencias Sociales, Niñez y Juventud, 15 (2), pp. 927-949. DOI:10.11600/1692715x.1520929042016.

Ramos, D. (2020). Representaciones sociales del paisaje hídrico en Fómeque, veredas Hato Viejo y Mortiñal. [Tesis de pregrado, Universidad Santo Tomás]. https://repository.usta.edu.co/handle/11634/29149.

Rengifo, B; Quitiaquez, L. y Mora, F. (2012). La educación ambiental una estrategia pedagógica que contribuye a la solución de la problemática ambiental en Colombia. XII Coloquio internacional de geocrítica. Recuperado de: http://www.ub.edu/geocrit/coloquio2012/actas/06-B-Rengifo.pdf.

Reyes, H., y Cardona, L. C. (2015). La educación ambiental como estrategia necesaria para la planificación de nuevos enfoques regionales en el departamento del Meta. Sophia, 11(2), 169-184.

Rivarosa, A., \& Perales, F. J. (2006). La resolución de problemas ambientales en la escuela y en la formación inicial de maestros. Revista Iberoamericana De Educación, 40, 111-124. https://doi.org/10.35362/rie400785.

Sauvé, L. (2010). Educación científica y educación ambiental: un cruce fecundo. Enseñanza de las ciencias. Revista de investigación y experiencias didácticas, 28(1), 5-18.

Sánchez, R. y Ossa, C. (2020). Environmental education in the Chilean school: pedagogical experiences in the commune of Santa Barbara. Revista Académica de la Universidad Católica del Maule, 59, 103-128. DOI: 10.29035/ucmaule.59.103.

Shiva, V. (1993). Monocultures of the mind. Trumpeter. ISBN-13: 978-1856492188. 
Tejeda, C. (2002). Apropiación social del territorio y política ambiental en la Selva Lacandona, Chiapas; el caso de Frontera Corozal, Comunidad Lacandona. Tesis de Maestría en Ciencias en Desarrollo Rural Regional. Dirección de Centros Regionales. Universidad Autónoma Chapingo. 